\title{
Deformation and spallation of a magnesium alloy under high strain rate loading
}

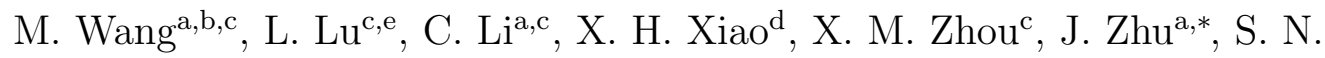 \\ $\mathrm{Luo}^{\mathrm{b}, \mathrm{c}, *}$ \\ ${ }^{a}$ College of Physical Science and Technology, Sichuan University, Chengdu, Sichuan \\ 610064, P. R. China \\ ${ }^{b}$ Key Laboratory of Advanced Technologies of Materials, Ministry of Education, \\ Southwest Jiaotong University, Chengdu, Sichuan 610031, P. R. China \\ ${ }^{c}$ The Peac Institute of Multiscale Sciences, Chengdu, Sichuan 610031, P. R. China \\ ${ }^{d}$ Advanced Photon Source, Argonne National Laboratory, Argonne, IL 60439, USA \\ ${ }^{e}$ CAS Key Laboratory of Mechanical Behavior and Design of Materials, Department of \\ Modern Mechanics, University of Science and Technology of China, Hefei, Anhui \\ 230027, P. R. China
}

\section{Abstract}

We investigate deformation and damage of a magnesium alloy, AZ91, under high strain rate $\left(\sim 10^{5} \mathrm{~s}^{-1}\right)$ loading via planar impact. The soft-recovered specimens are examined with electron back-scatter diffraction (EBSD). EBSD analysis reveals three types of twinning: $\{10 \overline{1} 2\}$ extension, $\{10 \overline{1} 1\}$ contraction, and $\{10 \overline{1} 1\}-\{10 \overline{1} 2\}$ double twinning, and their number density increases with increasing impact velocity. The extension twins dominate contraction and double twins in size and number. Dislocation densities of the recovered specimens are evaluated with x-ray diffraction, and increase with increasing impact velocity. X-ray tomography is used to resolve three-dimensional microstructure of shock-recovered samples. The EBSD and tomography re-

\footnotetext{
*Corresponding author

Email addresses: zhujun01@163.com (J. Zhu), sluo@pims.ac.cn; sluo@swjtu.cn
} (S. N. Luo) 
sults demonstrate that the second phase, $\mathrm{Mg}_{17} \mathrm{Al}_{12}$, plays an important role in both deformation twinning and tensile cracking. Deformation twinning appears to be a common mechanism in deformation of magnesium alloys at low, medium and high strain rates, in addition to dislocation motion.

Keywords: Magnesium alloys, Twinning, Strain rate, Impact, EBSD

\section{Introduction}

Magnesium alloys show a remarkable potential for their low density, high specific strength, and high specific stiffness $[1,2]$. Normally used as die cast products (e.g., AZ91), they offer a combination of desirable mechanical properties, castability and corrosion resistance $[3,4]$. During service, magnesium alloys may be subjected to impulsive loading such as impact, and their dynamic responses under high strain rate loading have been investigated with split Hopkinson compression or tension bars $\left(10^{2}-10^{4} \mathrm{~s}^{-1}\right)[5,6,7,8,9]$, and to a lesser extent, with gas gun plate impact at higher strain rates $\left(\sim 10^{5} \mathrm{~s}^{-1}\right.$ or higher) $[10,11,12,13,14,15]$.

Under one-dimensional (1D) strain shock loading, dynamic tensile strength or spall strength of Mg95 (99.95 wt.\% Mg) was studied over a wide range of temperatures [10]. The shear strength of magnesium alloy AZ61 was observed to increase with increasing peak shock stress [12]. The effects of orientation and heat treatment on the Hugoniot elastic limit and spall strength of magnesium alloy Elektron 675 were examined, and their values are higher along the extrusion direction [13]. The elastic precursor decay in magnesium alloy Ma2-1 under shock compression was explored for different sample thicknesses [14]. Such planar impact studies provide useful information on bulk mechan- 
ical properties of magnesium alloys. However, shock-induced microstructure changes and deformation mechanisms are rarely addressed, despite their apparent importance for high strain rate applications.

In cast magnesium alloy AZ91, the main strengthening intermetallic (the second phase) is $\beta-\mathrm{Mg}_{17} \mathrm{Al}_{12}$ dispersed within grains or at grain boundaries. This body-centered cubic structure of $\mathrm{Mg}_{17} \mathrm{Al}_{12}$ is incompatible with the close-packed hexagonal (HCP) structure of the magnesium matrix. In Hopkinson bar tests, the $\mathrm{Mg} / \mathrm{Mg}_{17} \mathrm{Al}_{12}$ interface acts as the crack initiation site in AZ91 alloy [7]; increasing the volume fraction of $\beta-\mathrm{Mg}_{17} \mathrm{Al}_{12}$ leads to an increase in yield strength and strain hardening, but a decrease in ductility and the twin fraction in AZ magnesium alloys under compression [16].

Deformation twinning modes in magnesium include $\{10 \overline{1} 2\}$ extension twinning, $\{10 \overline{1} 1\}$ contraction twinning $[17,18]$, and $\{10 \overline{1} 1\}-\{10 \overline{1} 2\}$ double twinning $[6,7,19,20,21,22,23,24,25]$, in the order of increasing difficulty in activation. It is the easiest to activate $\{10 \overline{1} 2\}$ extension twinning at various strain rates, and $\{10 \overline{1} 1\}$ contraction twinning is observed in $c$-axis compression of pure magnesium single crystals and polycrystalline magnesium alloys [17, 26, 27]. Contraction twinning and double twinning are often observed in uniaxial tension, while extension twinning prevails in uniaxial compression and ring hoop tension testing [20]. $\{10 \overline{1} 1\}-\{10 \overline{1} 2\}$ double twinning is responsible for shear failure of magnesium alloy AZ31 at room temperature, and yet $\{10 \overline{1} 2\}$ extension twinning has little influence on void formation [22]. Hopkinson bar tests revealed that increasing strain rate $\left(\sim 10^{3} \mathrm{~s}^{-1}\right)$ enhances the activation of $\{10 \overline{1} 2\}$ extension twinning dramatically, while its effects on contraction and double twinning are insignificant [6]. 
At high strain rates comparable to gas gun loading $\left(10^{4}-10^{7} \mathrm{~s}^{-1}\right)$, few experiments have investigated deformation mechanisms of magnesium alloys in general. In this work, we explore high strain rate deformation of magnesium alloy AZ91 with gas-gun plate impact loading at different impact velocities $\left(170-265 \mathrm{~m} \mathrm{~s}^{-1}\right)$. The shock-recovered samples are examined with electron backscatter diffraction (EBSD), x-ray tomography and diffraction. While $\{10 \overline{1} 2\}$ extension twinning still dominates deformation, $\{10 \overline{1} 1\}$ contraction twinning and $\{10 \overline{1} 1\}-\{10 \overline{1} 2\}$ double twinning also occur at higher impact velocities. The number density of twins and dislocation density increase with increasing impact velocity. The second phase, $\mathrm{Mg}_{17} \mathrm{Al}_{12}$, plays an important role in both deformation twinning and tensile cracking.

\section{Materials and experiments}

The cast magnesium alloy AZ91 in this work contains $9.0 \mathrm{wt} \% \mathrm{Al}, 0.6$ wt\% Zn, 0.4 wt\% Mn, and 90.0 wt\% Mg. The initial microstructure is analyzed with EBSD (Fig. 1(a)). Its grain size ranges from $100 \mu \mathrm{m}$ to $500 \mu \mathrm{m}$ with abundant $\beta-\mathrm{Mg}_{17} \mathrm{Al}_{12}$ phase distributed "randomly" in grain interiors or at grain boundaries. Both flyer plates and samples have a diameter of 10 $\mathrm{mm}$ and are made of the same batch of AZ91 for symmetric impact. The thicknesses of flyer plates and samples are $0.7 \mathrm{~mm}$ and $1.4 \mathrm{~mm}$ respectively. Two parallel surfaces of a target or flyer plate are polished to micron level or mirror finish.

Flyer plate planar impact experiments are conducted with a $10-\mathrm{mm}$ bore, single-stage gas gun to investigate dynamic tension response of the AZ91 samples initially at room temperature. Figure 1(b) shows the schematic 
setup of flyer plate impact experiments. A flyer plate (5) is attached to polycarbonate sabot (3), with a recess (4) immediately behind it. When a solenoid valve is fired, compressed nitrogen is released from a high-pressure gas reservoir into the gun barrel (1), accelerating the sabot and flyer plate assembly. Upon exiting the muzzle, the flyer plate impacts the target or sample (8) under consideration. The flyer plate velocity is measured with an optical beam block system (6). The muzzle and sample are located in a vacuum chamber (10). The shocked samples are "soft" recovered with foams (9) for EBSD, x-ray tomography and diffraction examination.

\section{Results and discussion}

Spallation experiments are conducted at three different impact velocities, $171 \mathrm{~m} \mathrm{~s}^{-1}, 218 \mathrm{~ms}^{-1}$ and $265 \mathrm{~ms}^{-1}$. When the release fans initiated from the back of the flyer plate and the sample free surface encounter and interact in the midsection of the target, this region undergoes a transition from compression to tension. Once the tensile stress exceeds a critical stress, termed as the spall strength, spall occurs. The methodology as well as data analysis related to planar impact experiments can be found in previous studies $[28,29,30,31]$.

During shock loading, there is a shock heating effect. However, the impact velocities are low (the maximum is $265 \mathrm{~m} \mathrm{~s}^{-1}$ ) and shock durations are short ( 300 ns). While dynamic recovery of microstructure due to shock heating does exist, it is expected to be small in our experimental conditions. In order to investigate shock-induced microstructure changes of the AZ91 magnesium alloy, the shock-recovered targets are sectioned into halves along the impact 
direction, and EBSD is utilized to analyze the cross-sections. EBSD inverse pole figures and the corresponding image quality maps of the samples deformed at various impact velocities are shown in Fig. 2. In addition, cracks are observed at grain boundaries and grain boundary triple junctions in the sample impacted at $265 \mathrm{~m} \mathrm{~s}^{-1}$ (Fig. 2(f)), while no obvious cracks are found for lower impact velocities. The second phase tend to conglomerate around grain boundaries, and both are weak spots for tensile microcrack nucleation. Consistently, cracks were found to be prone to nucleate at the $\mathrm{Mg} / \mathrm{Mg}_{17} \mathrm{Al}_{12}$ interfaces [16]. The growth and coalescence of microcracks lead to intergranular cracking.

X-ray tomography is used to investigate microstructure of the spallation region in the shock-recovered specimens. X-ray tomography is a nondestructive 3D technique capable of resolving internal structure with micron resolutions, and conducted at the 2-BM beamline of the Advanced Photon Source. The 3D microstructure reconstructed with tomography is shown in Fig. 3 for the specimen impacted at $265 \mathrm{~m} \mathrm{~s}^{-1}$, showing three structural components: the magnesium matrix, the second phase $\left(\mathrm{Mg}_{17} \mathrm{Al}_{12}\right)$, and cracks. The second phase is distributed inhomogeneously, consistent with the EBSD result. Numerous cracks are induced during tension and most of them are surrounded by the second phase, indicating that the interfaces between the second phase and the matrix are the nucleation sites for cracks.

All the regions indicated in Fig. 2 are located in the mid-sections of the targets, which are subjected to tension and possible spall. Under high strain rate tension, the most pronounced deformation feature is reorientation caused by twinning. The distribution of deformation twins is non-uniform, 
with some twins clustering around the $\beta$-phases (marked with black arrows in Fig. 2). The twins are mostly lens-shaped, thin and short at low impact velocities (171 $\mathrm{m} \mathrm{s}^{-1}$; Fig. 2(a)), and grow in size or number density of at higher impact velocities (Figs. 2(c) and 2(e)).

To quantify the microstructure features of AZ91 induced by impact, we calculate the area fraction of all twins over the total EBSD sampling area, the area fraction of the twins connected with the second phase $(\beta)$ over the total twin area, and number density of all twins (Fig. 4(a)). Both twin area fractions and number density increase with increasing impact velocity approximately in a linear manner. The total twin area fraction increases from $\sim 3 \%$ to $22 \%$, the twins intersecting with the second phase, or simply, the second-phase twins, occupy $12 \%$ to $25 \%$ of the total twin areas, and the number density of all the twins rises from about 1000 to $2000 \mathrm{~mm}^{-2}$. The twins connected with the second phase are likely initiated from the phase boundaries due to reduced energy barrier to nucleation and dislocation pileups. The second phase acts as nucleation sites and the pinning points during twin growth.

Three types of deformation twinning in magnesium alloys are reported: $\{10 \overline{1} 2\}$ extension, $\{10 \overline{1} 1\}$ contraction and $\{10 \overline{1} 1\}-\{10 \overline{1} 2\}$ double twinning $[7,24,25]$. A $\{10 \overline{1} 2\}$ extension twin is activated when an extension strain is applied parallel to the $c$-axis or a contraction strain is applied perpendicular to the $c$-axis, while a $\{10 \overline{1} 1\}$ contraction twin is activated when there is a contraction strain component parallel to the $c$-axis or when extension is applied normal to the $c$-axis. For double twins, a primary $\{10 \overline{1} 1\}$ contraction twin forms first, and then the secondary $\{10 \overline{1} 2\}$ twin develops within the 
$\{10 \overline{1} 1\}$ contraction twin, leading to contraction along the $c$-axis. The basal planes in the twin and those in the matrix form an angle of $86.3^{\circ}, 56.2^{\circ}$ and $37.5^{\circ}$ for $\{10 \overline{1} 2\}$ extension, $\{10 \overline{1} 1\}$ contraction and $\{10 \overline{1} 1\}-\{10 \overline{1} 2\}$ double twins, respectively, as illustrated in Fig. 5.

Different types of deformation twins formed during impact loading of AZ91 are depicted with different colors in the image quality maps (the right column, Fig. 2). At a low impact velocity $\left(171 \mathrm{~m} \mathrm{~s}^{-1}\right)$, only $\{10 \overline{1} 2\}$ extension twins form. With increasing impact velocity, $\{10 \overline{1} 1\}$ contraction twinning and $\{10 \overline{1} 1\}-\{10 \overline{1} 2\}$ double twining also occur in addition to extension twinning; and all the twins increase in number or size. The fractions of contraction and double twins over all the twins are less than $1 \%$ even at the highest impact velocity. Fig. 4(b) shows the neighbor-to-neighbor misorientation angle distributions measured at different impact velocities. The misorientation angle distribution is dominated by a high frequency peak in the range of $85-90^{\circ}$ in all shock-recovered samples, which corresponds to numerous $\{10 \overline{1} 2\}$ extension twin boundaries $\left(86.3^{\circ}\right.$, Fig. $\left.5(\mathrm{a})\right)$ presented in the image quality maps. Additionally, a modest rise in the misorientation angle at $55-60^{\circ}$ is also observed with increasing impact velocity, indicating a number density increase in $\{10 \overline{1} 1\}$ contraction twins $\left(56.2^{\circ}\right.$, Fig. $\left.5(\mathrm{~b})\right)$. Double twins are not shown in Fig. 4(b) since their number density is minor compared to extension and contraction twins. The decrease in abundances corresponds to an increase in the critical resolved shear stress (CRSS), although other factors besides CRSS could also affect twinning. CRSS measured experimentally for $\{10 \overline{1} 2\}$ extension twins is about $2-5 \mathrm{MPa}$, which is much smaller than the value for $\{10 \overline{1} 1\}$ contraction twins $(30-100 \mathrm{MPa})$. Thus, activation of a $\{10 \overline{1} 2\}$ 
contraction twin is difficult due to its high CRSS, and it is even more difficult to activate $\{10 \overline{1} 1\}-\{10 \overline{1} 2\}$ double twins since they have to originate within contraction twin interiors.

Besides deformation twinning, dislocation motion also plays an important role in plastic deformation. The shock-recovered samples are analyzed with x-ray diffraction using a Panalytical X'pert microfocus x-ray diffractometer with $\mathrm{Cu} K_{\alpha}$ radiation $(1.54056 \AA)$. The x-ray spot, $300 \mu \mathrm{m}$ in diameter, illuminates the tensile region near the spallation plane (in the middle of the cross-section) where deformation twins form under tension. Given the large grain sizes, the "microfocus" diffraction profiles are similar to those of single crystals. The measured (10̄11) diffraction peaks (Fig. 6) show increasing peak broadening with increasing impact velocity, indicating increasing dislocation activities. The peak profiles are evaluated to obtain dislocation densities, and the methodology is described in detail in Ref. [32]. The dislocation densities are approximately $0.8 \times 10^{14}, 1.0 \times 10^{14}$, and $1.7 \times 10^{14} \mathrm{~m}^{-2}$, increasing with increasing impact velocity. The dislocation density for the as-received (unshocked) sample $\left(0.8 \times 10^{14} \mathrm{~m}^{-2}\right)$ is similar to the values reported in literature [33].

Under high strain rate flyer plate impact, numerous $\{10 \overline{1} 2\}$ extension twins are observed in AZ91, similar to the observations from quasi-static or Hopkinson compression/tension bar experiments. In previous Hopkinson bar tests (1D stress loading with $100 \mu$ s-level pulse durations at $10^{3} \mathrm{~s}^{-1}$ ) on finegrained samples $(10 \mu \mathrm{m})$, increasing strain rate enhances the activation of $\{10 \overline{1} 2\}$ extension twinning dramatically [6]. Similarly, the number density of $\{10 \overline{1} 2\}$ extension twin shows a distinct increase with increasing impact ve- 
locity under shock as shown in Fig. 4. Furthermore, the $\{10 \overline{1} 1\}$ contraction and $\{10 \overline{1} 1\}-\{10 \overline{1} 2\}$ double twins are activated only when AZ91 is subjected to higher shock-induced tension loading, and their number densities undergo a moderate increase. However, the contraction twinning and double twinning in magnesium alloy reported by Dudamell et al. are insensitive to strain rates [6]. The discrepancy could be due to the drastic differences in loading conditions (1D stress vs $1 \mathrm{D}$ strain), loading pulse durations (100 $\mu$ s vs $300 \mathrm{~ns})$, and grain sizes $(10 \mu \mathrm{m}$ vs. $100 \mu \mathrm{m})$. Our work with a first analysis of microstructure changes induced by high strain rate loading, together with previous microstructure studies at low and medium strain rates, suggest that deformation twinning is the main, common, mechanism in deformation of magnesium alloys at low, medium and high strain rates. However, future work should explore systematically the effects of exact stress states (1D stress vs. 1D strain, for instance), stress history, grain size, texture, and compositions.

\section{Conclusions}

In summary, flyer plate impact experiments with various impact velocities are conducted to investigate dynamic response of a magnesium alloy, AZ91. The shock-recovered samples are examined with EBSD to analyze the microstructure changes induced by strain rate loading. $\{10 \overline{1} 2\}$ extension twining is the predominant twinning mechanism regardless of impact velocity, while $\{10 \overline{1} 1\}$ contraction twinning and $\{10 \overline{1} 1\}-\{10 \overline{1} 2\}$ double twinning are activated at higher impact velocities. With increasing impact velocity, these twins increase in size and number, and dislocation density increases as 
well. The second phase, $\mathrm{Mg}_{17} \mathrm{Al}_{12}$, plays an important role in both deformation twinning and intergranular cracking. Deformation twinning appears to be the main, common, mechanism in deformation of magnesium alloys at low, medium and high strain rates.

\section{Acknowledgments}

Use of the Advanced Photon Source, an Office of Science User Facility operated for the (U.S.) Department of Energy (DOE) Office of Science by Argonne National Laboratory, was supported by the (U.S.) DOE under Contract No. DE-AC02-06CH11357.

\section{References}

[1] B. L. Mordike, T. Ebert, Mater. Sci. Eng. A 302 (2001) 37-45.

[2] M. Easton, A. Beer, M. Barnett, C. Davies, G. Dunlop, Y. Durandet, S. Blacket, T. Hilditch, P. Beggs, JOM 60 (2008) 57-62.

[3] I. J. Polmear, Mater. Sci. Tech. 10 (1994) 1-16.

[4] Z. Zhang, A. Couture, A. Luo, Scripta Mater. 39 (1998) 45-53.

[5] M. T. Tucker, M. F. Horstemeyer, P. M. Gullett, H. E. Kadiri, W. R. Whittington, Scripta Mater. 60 (2009) 182-185.

[6] N. Dudamell, I. Ulacia, F. Gálvez, S. Yi, J. Bohlen, D. Letzig, I. Hurtado, M. T. Pérez-Prado, Acta Mater. 59 (2011) 6949-6962.

[7] H. Asgari, J. A. Szpunar, A. G. Odeshi, Mate. Design 61 (2014) 26-34. 
[8] K. E. Prasad, B. Li, N. Dixit, M. Shaffer, S. Mathaudhu, K. T. Ramesh, JOM 66 (2014) 291-304.

[9] N. Dixit, K. Y. Xie, K. J. Hemker, K. T. Ramesh, Acta Mater. 87 (2015) $56-67$.

[10] G. I. Kanel, S. V. Razorenov, A. Bogatch, A. V. Utkin, V. E. Fortov, D. E. Grady, J. Appl. Phys. 79 (1996) 8310-8317.

[11] G. I. Kanel, S. V. Razorenov, A. Bogatch, A. V. Utkin, D. E. Grady, Int. J. Impact Eng. 20 (1997) 467-478.

[12] J. C. F. Millett, S. M. Stirk, N. K. Bourne, G. T. Gray, Acta Mater. 58 (2010) 5675-5682.

[13] P. J. Hazell, G. J. Appleby-Thomas, E. Wielewski, C. Stennett, C. Siviour, Acta Mater. 60 (2012) 6042-6050.

[14] G. V. Garkushin, G. I. Kanel, S. V. Razorenov, Phys. Solid State 54 (2012) 1079-1085.

[15] P. J. Hazell, G. J. Appleby-Thomas, E. Wielewski, J. P. Escobedo, Philos. T. R. Soc. A 372 (2014) 20130204.

[16] Y. Z. Lü, Q. D. Wang, W. J. Ding, X. Q. Zeng, Y. P. Zhu, Mater. Lett. 44 (2000) 265-268.

[17] M. R. Barnett, Mater. Sci. Eng. A 464 (2007) 8-16.

[18] N. Stanford, M. Barnett, Scripta Mater. 58 (2008) 179-182.

[19] P. Cizek, M. R. Barnett, Scripta Mater. 59 (2008) 959-962. 
[20] L. Jiang, J. J. Jonas, R. Mishra, A. Luo, A. Sachdev, S. Godet, Acta Mater. 55 (2007) 3899-3910.

[21] A. Jain, O. Duygulu, D. Brown, C. Tomé, S. Agnew, Mater. Sci. Eng. A 486 (2008) 545-555.

[22] T. Al-Samman, G. Gottstein, Mater. Sci. Eng. A 488 (2008) 406-414.

[23] Y. Xin, M. Wang, Z. Zeng, M. Nie, Q. Liu, Scripta Mater. 66 (2012) 25-28.

[24] M. R. Barnett, Z. Keshavarz, A. G. Beer, X. Ma, Acta Mater. 56 (2008) 5-15.

[25] S. W. Xu, S. Kamado, N. Matsumoto, T. Honma, Y. Kojima, Mater. Sci. Eng. A 527 (2009) 52-60.

[26] M. D. Nave, M. R. Barnett, Scripta Mater. 51 (2004) 881-885.

[27] Z. Keshavarz, M. R. Barnett, Scripta Mater. 55 (2006) 915-918.

[28] M. A. Meyers, Dynamic behavior of materials, John Wiley \& Sons, New York, 1994.

[29] S. N. Luo, Q. An, T. C. Germann, L. B. Han, J. Appl. Phys. 106 (2009) 013502.

[30] B. X. Bie, J. H. Han, L. Lu, X. M. Zhou, M. L. Qi, Z. Zhang, S. N. Luo, Compos. Part A-Appl. S. 68 (2015) 282-288. 
[31] T. Li, D. Fan, L. Lu, J. Y. Huang, J. C. E, F. Zhao, M. L. Qi, T. Sun, K. Fezzaa, X. H. Xiao, X. M. Zhou, T. Suo, W. Chen, Y. L. Li, M. H. Zhu, S. N. Luo, Carbon 91 (2015) 468-478.

[32] M. Wilkens, Phys. Status Solidi A 2 (1970) 359-370.

[33] K. Máthis, J. Gubicza, N. Nam, J. Alloy Compd. 394 (2005) 194-199. 


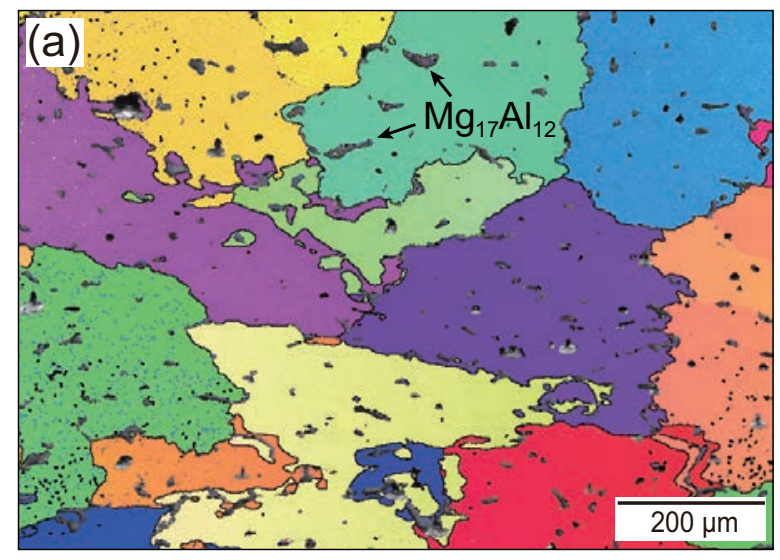

(b)

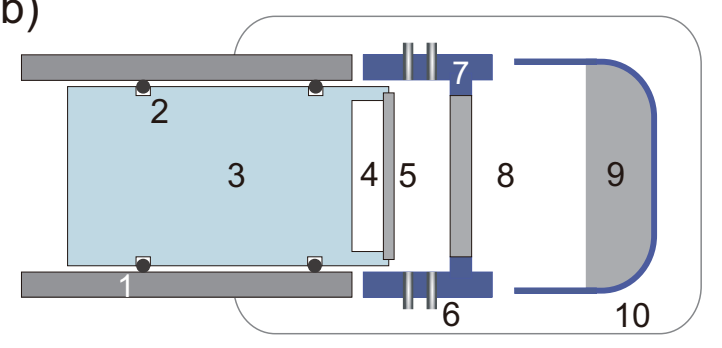

Figure 1: (a) EBSD orientation map of an as-received AZ91 specimen. (b) Schematic setup of gas-gun plate-impact experiments. 1: gun barrel; 2: O-ring; 3: polycarbonate sabot; 4: recess for release waves; 5: flyer plate; 6 : optical fibers and detectors for the optical beam block system; 7: sample holder; 8: sample; 9: soft materials; 10: vacuum chamber. 

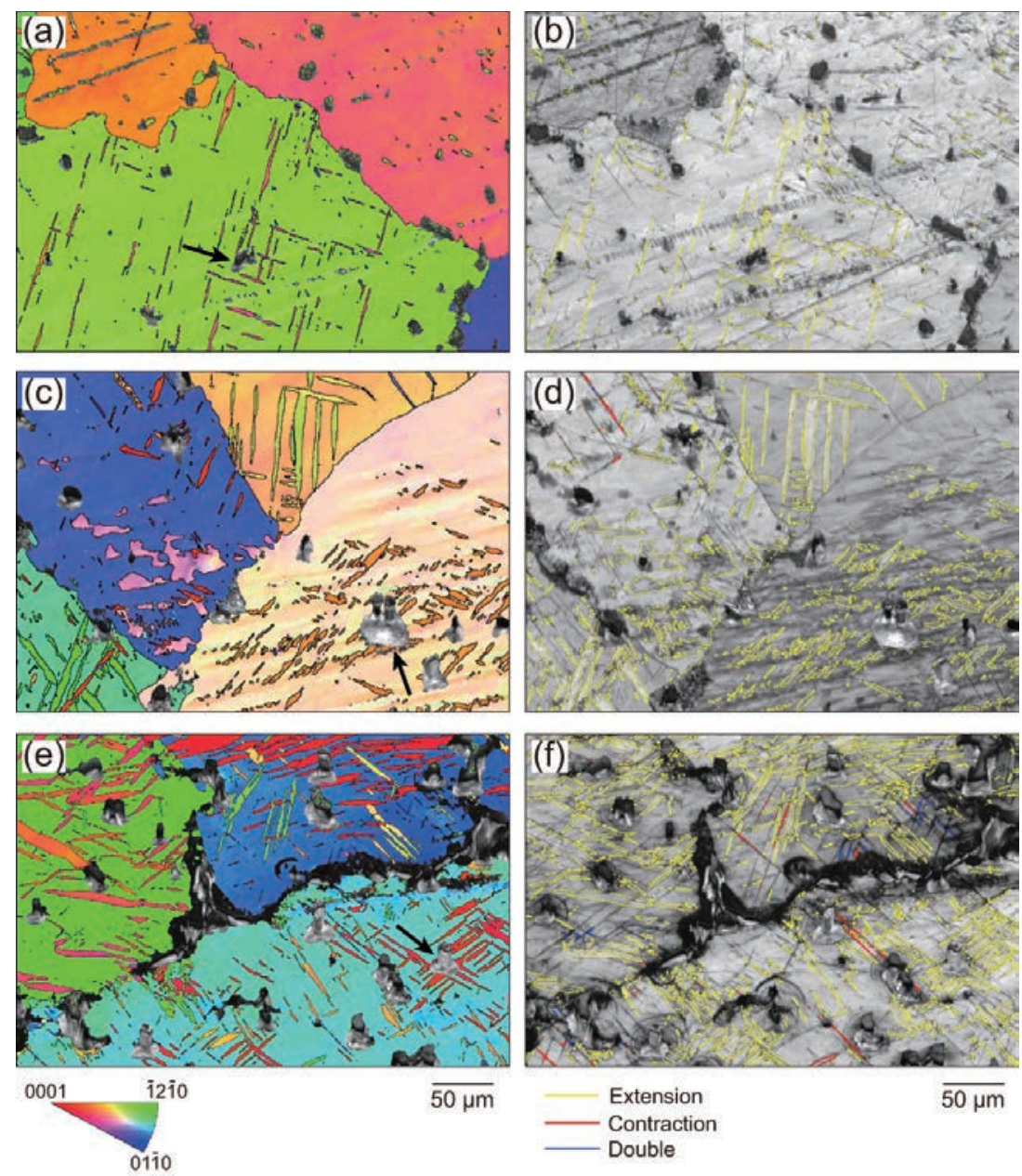

Figure 2: Cross-sectional EBSD inverse pole figure (IPF) maps (left column) of the shockrecovered samples, and the corresponding image quality maps overlaid with twin boundaries (right column). The impact velocity is $171 \mathrm{~m} \mathrm{~s}^{-1}$ for (a) and (b), $218 \mathrm{~m} \mathrm{~s}^{-1}$ for (c) and (d), and $265 \mathrm{~m} \mathrm{~s}^{-1}$ for (e) and (f). Arrows in the left column denote the second phase $\mathrm{Mg}_{17} \mathrm{Al}_{12}$. In the right column, yellow curves refers to extension twin boundaries $\left(86.3^{\circ}\langle 1 \overline{2} 10\rangle \pm 5^{\circ}\right)$, red curves to contraction twin boundaries $\left(56.2^{\circ}\langle 1 \overline{2} 10\rangle \pm 5^{\circ}\right)$, and blue curves to double twin boundaries $\left(37.5^{\circ}\langle 1 \overline{2} 10\rangle \pm 5^{\circ}\right)$. 


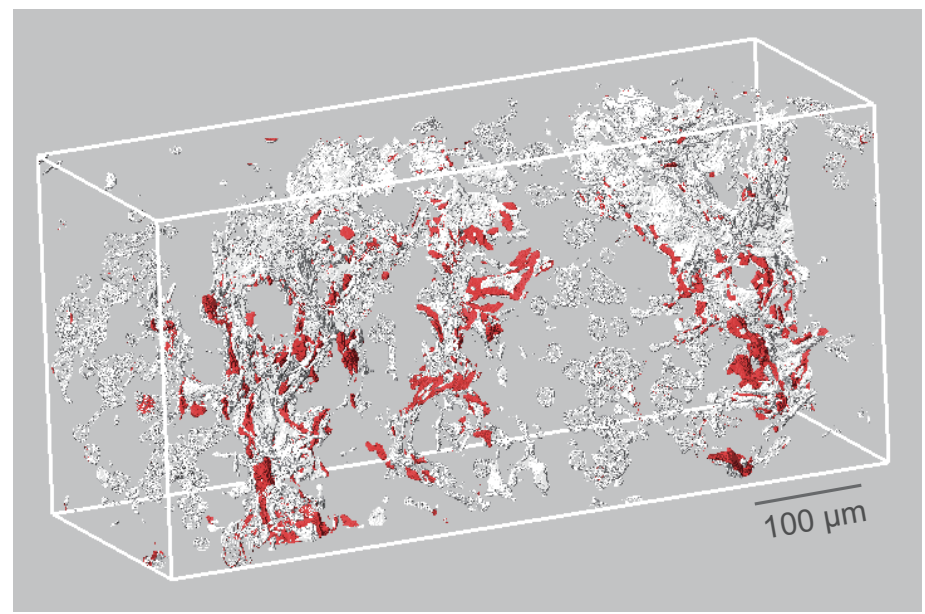

Figure 3: X-ray tomography characterization of spallation region in the soft-received sample impacted at a velocity of $265 \mathrm{~m} \mathrm{~s}^{-1}$. Red donates cracks, and white represents the second phase. The magnesium matrix is not shown for clarity. 


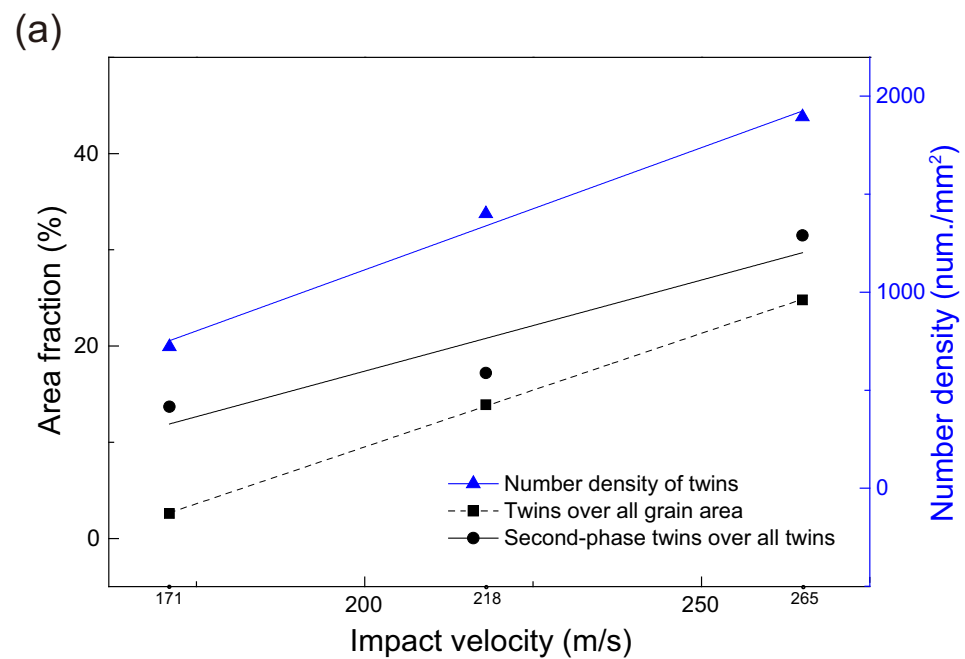

(b)

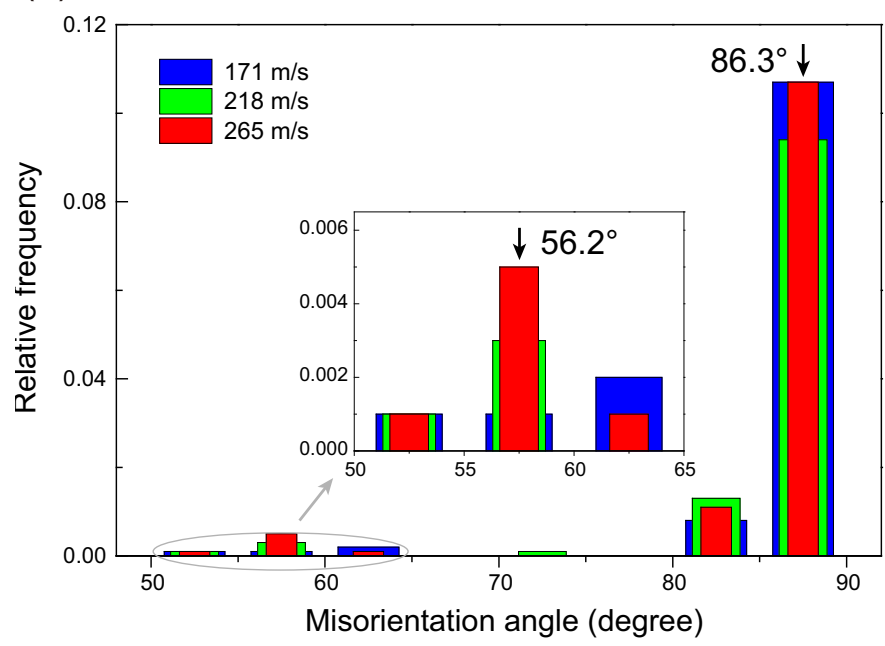

Figure 4: Statistics of twins. (a) Area fraction of all twins over all the EBSD sampling area (dots), second-phase twin area fraction over the total twin area (squares), and number density of all twins (triangles) as a function of impact velocity. Straight lines are the guide to the eyes. (b) Neighbour-to-neighbour misorientation angle distributions for three impact velocities (corresponding to Fig. 2). 
(a)

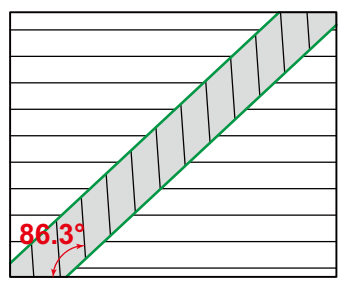

$\{10 \overline{1} 2\}$ (b)

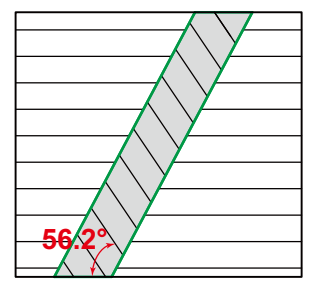

$\{10 \overline{1} 1\}$ (c)

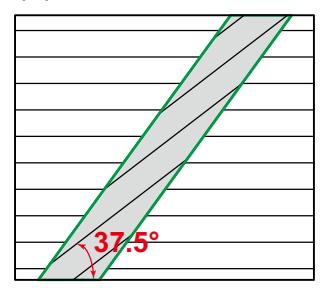

$\{10 \overline{1} 1\}-\{10 \overline{1} 2\}$

Figure 5: Schematic configurations of three types of deformation twins. (a) $\{10 \overline{1} 2\}$ extension twin. (b) $\{10 \overline{1} 1\}$ contraction twin. (c) $\{10 \overline{1} 1\}-\{10 \overline{1} 2\}$ double twin. The crosshatching lines correspond to the basal plane and the image zone axis is $\langle 1 \overline{2} 10\rangle$.

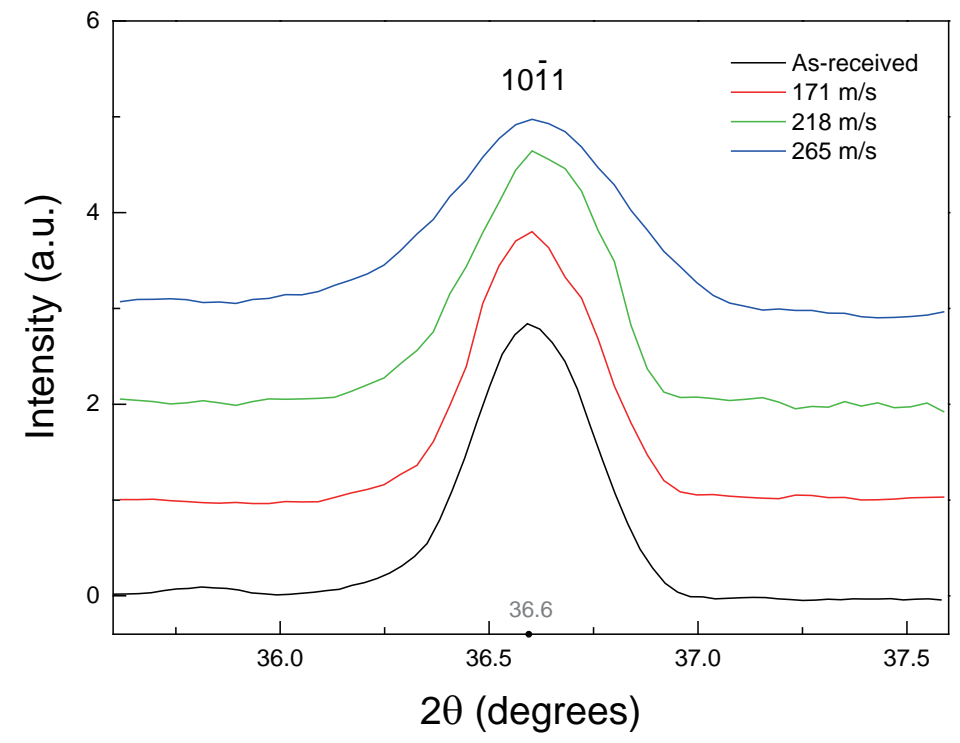

Figure 6: Normalized XRD profiles of (1011) reflections for the as-received and shockrecovered specimens. The baselines are shifted for clarity. 